\title{
Global Existence and Uniqueness of Solutions for a Free Boundary Problem Modeling the Growth of Tumors with a Necrotic Core and a Time Delay in Process of Proliferation
}

\author{
Shihe $\mathrm{Xu}$ and Minhai Huang \\ School of Mathematics and Statistics, Zhaoqing University, Zhaoqing, Guangdong 526061, China \\ Correspondence should be addressed to Shihe Xu; shihe56789@163.com \\ Received 7 March 2014; Revised 29 April 2014; Accepted 11 May 2014; Published 29 May 2014 \\ Academic Editor: Hak-Keung Lam \\ Copyright (C) 2014 S. Xu and M. Huang. This is an open access article distributed under the Creative Commons Attribution License, \\ which permits unrestricted use, distribution, and reproduction in any medium, provided the original work is properly cited.

\begin{abstract}
We study a mathematical model for the growth of necrotic tumors with time delays in proliferation. By transforming this problem into an initial-boundary value problem in fixed domain of a coupled system of a parabolic equation and one integrodifferential equation with time delays, in which all equations involve discontinuous terms, and using the approximation method combined with Schauder fixed point theorem, we prove that this problem has a unique global solution in any time interval $[0, T]$.
\end{abstract}

\section{Introduction}

Tumor is a major threat in public health; it can cause serious problems for people at all ages. However, tumor progression is a complex process. The understanding of its dynamics is one of the great challenges of modern medical science. To describe the growth of solid tumors, an increasing number of mathematical models in forms of free boundary problems of partial differential equations have been proposed and studied during the past several decades (see, e.g., [1-12] and the references cited therein). Most of those models are based on the reaction-diffusion equations and mass conservation law. Analysis of such mathematical models has drawn great interest, and many results have been established; compare [13-22] and references therein. Many numerical results have been performed; compare $[1,2,13,23,24]$ and references therein. Analysis of such models can help us to examine and distinguish different functions of different mechanisms involved in tumor-growth process and may also assist us in assessing effects of various drug treatments and chemotherapy.

The first equation of the model which we will study in this paper is a reaction-diffusion equation for the nutrient concentration $\sigma(r, t)$, which we present in dimensionless form:

$$
\begin{array}{r}
c \frac{\partial \sigma}{\partial t}=\frac{1}{r^{2}} \frac{\partial}{\partial r}\left(r^{2} \frac{\partial \sigma}{\partial r}\right)-F(\sigma) H\left(\sigma-\sigma_{N}\right), \\
0<r<R(t), \quad t>0,
\end{array}
$$

where $H$ is the Heaviside function and $c$ represents the time scale of diffusion of nutrient compared to the time scale of the tumor doubling within the tumor. $F(\sigma)$ in (1) is the consumption rate of nutrient in a unit volume in the region $\rho(t)<r<R(t), t>0$; (1) means dead cells do not consume the nutrient. $\sigma_{N}$ is the minimal nutrient concentration needed for proliferation; that is, $\sigma_{N}$ is a positive constant representing a threshold value for necrosis. In the region where $\sigma>\sigma_{N}$, nutrient is enough to sustain (at least a portion of) tumor cells alive, whereas in the region where $\sigma \leq \sigma_{N}$, nutrient is insufficient to sustain any tumor cells alive and all cells there are dead cells.

Let $d=(1 / c)$ and $F(\sigma)=c f(\sigma)$; we have

$$
\begin{aligned}
\frac{\partial \sigma}{\partial t}=d \frac{1}{r^{2}} \frac{\partial}{\partial r}\left(r^{2} \frac{\partial \sigma}{\partial r}\right) & -f(\sigma) H\left(\sigma-\sigma_{N}\right), \\
0 & <r<R(t), \quad t>0 .
\end{aligned}
$$


The initial and boundary conditions for the necrotic phase of the tumor are

$$
\begin{gathered}
\frac{\partial \sigma}{\partial r}(0, t)=0, \quad \sigma(R(t), t)=\sigma_{\infty}, \quad t>0, \\
\frac{\partial \sigma}{\partial r}(\rho(t)-0, t)=\frac{\partial \sigma}{\partial r}(\rho(t)+0, t), \quad t>0, \\
\sigma(\rho(t)-0, t)=\sigma(\rho(t)+0, t)=\sigma_{N}, \quad t>0, \\
\sigma(r, t)=\psi(r, t), \quad 0 \leq r \leq R(t),-\tau \leq t \leq 0, \\
\rho(t)=\phi(t), \quad-\tau \leq t \leq 0, \\
R(t)=\varphi(t), \quad-\tau \leq t \leq 0 .
\end{gathered}
$$

The second equation of the model

$$
\begin{array}{r}
\frac{d R}{d t}=\frac{s}{R^{2}}\left(\int_{\rho(t-\tau)}^{R(t-\tau)} \sigma(r, t-\tau) r^{2} d r\right. \\
\left.-\int_{\rho(t)}^{R(t)} \tilde{\sigma} r^{2} d r-\int_{0}^{\rho(t)} \lambda r^{2} d r\right), \\
t>0,
\end{array}
$$

describes the evolution of the tumor radius $R(t)$ and is obtained by applying mass balance equations with adequate constitutive laws, where $R(t)$ and $\rho(t)$ denote the external and necrotic radius of tumor at time $t$, respectively, and $\sigma_{\infty}$ denotes the external concentration of nutrients, which is assumed to be a constant. The three terms on the right-hand side of (4) have the following explanation. The first term is the total tumor volume increase in a unit time interval induced by cell proliferation; the rate of cell proliferation in a unit volume is assumed to be proportional to $\sigma$. The second term is the total volume shrinkage in a unit time interval caused by cell apoptosis; the rate of cell apoptosis in a unit volume is assumed to be $s \widetilde{\sigma}$. The third term is the total volume shrinkage induced by dead cell dissolution; the rate of cell dissolution in a unit volume is assumed to be $s \lambda . s$ is a scaling constant.

Equations (1) and (3) are from Bueno et al. [15]. And (4) is from [1]. The process of tumor growth can be divided into several different stages, starting from the very early stage of solid tumor without necrotic core inside (cf. [19, $20,22,25,26])$. In the present paper we focus on the next stage, that is, the process of necrotic core formation. In this stage there are three main cellular processes: proliferation, apoptosis, and necrosis (cf. $[1,2,8,13,14,16,27,28]$ ). For process of necrotic core formation, the study by using the method of mathematical models was initiated by Byrne in [1]. Recently this study has drawn the attention of some other researchers; compare Bueno et al. [15], Cui [16], Foryś and Mokwa-Borkowska [13], and references cited therein. But all of them are related to quasistationary version; that is, $c=0$, and Cui [16] and Foryś and Mokwa-Borkowska [13] studied the particular cases in which $F(\sigma)=\Gamma \sigma$ and $F(\sigma)=a$, respectively, where $\Gamma$ and $a$ are two constants.

The model we studied in this paper is established by modifying the model studied in [15] by introducing a time delay in proliferation as that in Byrne [1]. In their model, the cell proliferation rate in unit volume is $S(\sigma)$, where $S$ is a continuous function which satisfies some conditions; that is, (4) is as follows $(\tau=0)$ :

$$
\begin{array}{r}
\frac{d R}{d t}=\frac{s}{R^{2}}\left(\int_{\rho(t)}^{R(t)} S(\sigma(r, t)) r^{2} d r\right. \\
\left.-\int_{\rho(t)}^{R(t)} \tilde{\sigma} r^{2} d r-\int_{0}^{\rho(t)} \lambda r^{2} d r\right), \\
t>0 .
\end{array}
$$

But for simplicity we assume that the cell proliferation rate in unit volume is proportional to its concentration; that is, $S(\sigma)=\mu \sigma$. The methods presented in this paper can be extended to the case that the cell proliferation rate in unit volume is $S(\sigma)$, where $S$ is a continuous function that satisfies some conditions. It should be pointed out that, in [15], the authors only study the existence and uniqueness of stationary solutions to the model (2)-(4), but in our study we mainly discuss the existence and uniqueness of the solution to the model (2)-(4).

\section{Main Results}

In this paper, we always use the following notation

$$
\begin{aligned}
& \bar{B}_{1}(0)=\left\{y \in R^{3},|y| \leq 1\right\}, \quad \bar{Q}_{T}=\bar{B}_{1}(0) \times[0, T], \\
& W_{p}^{2,1}\left(\bar{Q}_{T}\right)=\left\{u \in L^{p}\left(\bar{Q}_{T}\right): \nabla u, \nabla^{2} u, u_{t} \in L^{p}\left(\bar{Q}_{T}\right)\right\} .
\end{aligned}
$$

We will prove a global existence and uniqueness theorem for the problem (2)-(4) under the following assumptions:

$\left(A_{1}\right) f(\sigma)=\sigma f_{1}(\sigma)$, where $f_{1}$ is defined and Lipschitz is continuous on $[0, \infty) \times[0, \infty)$, and $f_{1}(\sigma) \geq 0$, for $\sigma \geq 0$

$\left(A_{2}\right) \varphi \in C[-\tau, 0], \varphi(0)>\rho(0)>0$, for $-\tau \leq t \leq$ $0, \psi \in C([0, \infty] \times[-\tau, 0])$, and $\psi(r, t)=\sigma_{\infty}$, for $r \geq$ $R(t) ; 0 \leq \psi(r, t) \leq \sigma_{\infty}$, for $r \leq R(t)$;

$\left(A_{3}\right) \psi(r, 0)=\psi_{0}(r)$ is twice weakly differentiable on $[0, R(0)], \psi_{0}^{\prime \prime} \in L^{\infty}[0, R(0)], \psi_{0}^{\prime}(0)=0, \psi_{0}(R(0))=$ $\sigma_{\infty}$

Our main result is as follows.

Theorem 1. Assume that conditions $\left(A_{1}\right),\left(A_{2}\right)$, and $\left(A_{3}\right)$ are satisfied. Then for any $T>0$, the problem (2)-(4) has a solution $(\sigma(r, t), R(t))$, for all $0 \leq t \leq T$, satisfying $\sigma(|x|, t) \in$ $W_{p}^{2,1}\left(Q_{T}\right)(5<p<\infty)$ and $0 \leq \sigma(|x|, t) \leq \sigma_{\infty}$.

\section{Transformation to a Fixed Domain}

We introduce a transformation of $(r, t, \sigma, R) \rightarrow(z, t, u, R)$ as follows:

$$
\begin{gathered}
z=\frac{r}{R(t)}, \quad t=t \\
u(z, t)=\sigma(z R(t), t), \quad R(t)=R(t) .
\end{gathered}
$$


Then the problem (2)-(4) is transformed into the following problem:

$$
\begin{aligned}
& \frac{\partial u}{\partial t}=d \frac{1}{R^{2}(t)} \frac{1}{z^{2}} \frac{\partial}{\partial z}\left(z^{2} \frac{\partial u}{\partial z}\right)+\frac{R^{\prime}(t)}{R(t)} z u_{z} \\
& -f(u) H\left(u-\sigma_{N}\right) \text {, } \\
& 0<z<1, t>0, \\
& \frac{\partial u}{\partial z}(0, t)=0, \quad u(1, t)=\sigma_{\infty}, \quad t>0 \\
& \frac{d R}{d t}=s R(t)\left(\int_{0}^{1} \frac{R_{\tau}^{3}}{R^{3}} u_{\tau} H\left(u_{\tau}-\sigma_{N}\right) z^{2}\right. \\
& -\widetilde{\sigma} H\left(u-\sigma_{N}\right) z^{2} \\
& \left.-\lambda\left[1-H\left(u-\sigma_{N}\right)\right] z^{2} d z\right), \\
& t>0 \text {, } \\
& u(z, t)=\psi(z R(t), t), \quad 0 \leq z \leq 1,-\tau \leq t \leq 0 \\
& \frac{\partial u}{\partial z}(z \rho(t)-0, t)=\frac{\partial u}{\partial z}(z \rho(t)+0, t), \\
& 0 \leq z \leq 1, \quad t>0, \\
& u(z \rho(t)-0, t)=u(z \rho(t)+0, t)=\sigma_{N}, \\
& 0 \leq z \leq 1, \quad t>0, \\
& \rho(t)=\phi(t), \quad-\tau \leq t \leq 0, \\
& R(t)=\varphi(t), \quad-\tau \leq t \leq 0,
\end{aligned}
$$

where $R_{\tau}=R(t-\tau), u_{\tau}=u(z, t-\tau)$. Let $X_{T}=C([0,1] \times$ $[0, T]) \times C([0,1] \times[-\tau, T])$. Denote the function $G$ as follows

$$
\begin{aligned}
G\left(u, u_{\tau}, t\right)=s\left(\int_{0}^{1}\right. & \frac{R_{\tau}^{3}}{R^{3}} u_{\tau} H\left(u_{\tau}-\sigma_{N}\right) z^{2} \\
& -\widetilde{\sigma} H\left(u-\sigma_{N}\right) z^{2} \\
& \left.-\lambda\left[1-H\left(u-\sigma_{N}\right)\right] z^{2} d z\right)
\end{aligned}
$$

for $t>0$. Using this notation, (10) can be simply written as follows:

$$
R^{\prime}(t)=R(t) G\left(u, u_{\tau}, t\right), \quad t>0 .
$$

It is clear that $R(t)$ can be expressed by

$$
R(t)=R_{0} \exp \left\{\int_{0}^{t} G\left(u, u_{\tau}, \xi\right) d \xi\right\}, \quad t>0 .
$$

Hence the problem (8)-(15) is reduced to the problem below:

$$
\begin{aligned}
& \frac{\partial u}{\partial t}=d R_{0}^{-2} \exp \left\{-2 \int_{0}^{t} G\left(u, u_{\tau}, \xi\right) d \xi\right\} \\
& \times \frac{1}{z^{2}} \frac{\partial}{\partial z}\left(z^{2} \frac{\partial u}{\partial z}\right)+G\left(u, u_{\tau}, t\right) z u_{z} \\
& -f(u) H\left(u-\sigma_{N}\right) \text {, } \\
& 0<z<1, \quad t>0 \text {, } \\
& \frac{\partial u}{\partial z}(0, t)=0, \quad u(1, t)=\sigma_{\infty}, \quad t>0, \\
& u(z, t)=\psi(z R(t), t), \quad 0 \leq z \leq 1,-\tau \leq t \leq 0, \\
& \frac{\partial u}{\partial z}(z \rho(t)-0, t)=\frac{\partial u}{\partial z}(z \rho(t)+0, t), \quad 0 \leq z \leq 1, t>0, \\
& u(z \rho(t)-0, t)=u(z \rho(t)+0, t)=\sigma_{N}, \quad 0 \leq z \leq 1, t>0, \\
& \begin{array}{ll}
\rho(t)=\phi(t), & -\tau \leq t \leq 0, \\
R(t)=\varphi(t), & -\tau \leq t \leq 0 .
\end{array}
\end{aligned}
$$

Then we conclude the following.

Lemma 2. Under the variable transformation (7), the free boundary problem (2)-(4) is equivalent to the initial-boundary value problem (19)-(25).

\section{The Proof of Main Results}

First we consider the following approximation problem:

$$
\begin{aligned}
\frac{\partial u}{\partial t}= & d R_{0}^{-2} \exp \left\{-2 \int_{0}^{t} G_{\varepsilon}\left(u, u_{\tau}, \xi\right) d \xi\right\} \\
& \times \frac{1}{z^{2}} \frac{\partial}{\partial z}\left(z^{2} \frac{\partial u}{\partial z}\right)+G_{\varepsilon}\left(u, u_{\tau}, t\right) z u_{z} \\
& -f(u) H\left(u-\sigma_{N}\right) \\
& 0<z<1, t>0
\end{aligned}
$$

with initial and boundary condition as (20)-(25), where $\varepsilon$ is an arbitrary positive number,

$$
\begin{aligned}
& G_{\varepsilon}\left(u, u_{\tau}, t\right)=s( \int_{0}^{1} \frac{R_{\tau}^{3}}{R^{3}} u_{\tau} H_{\varepsilon}\left(u_{\tau}-\sigma_{N}\right) z^{2} \\
&-\tilde{\sigma} H_{\varepsilon}\left(u-\sigma_{N}\right) z^{2} \\
&\left.-\lambda\left[1-H_{\varepsilon}\left(u-\sigma_{N}\right)\right] z^{2} d z\right), \\
& H_{\varepsilon}(x)= \begin{cases}1, & x \geq \varepsilon, \\
\frac{\varepsilon}{x}, & 0<x<\varepsilon, \\
0, & x \leq 0 .\end{cases}
\end{aligned}
$$

It is clear that $H_{\varepsilon}$ is a Lipschitz continuous function. 
Recall that $u(z, t)=\psi(z R(t), t), 0 \leq z \leq 1,-\tau \leq$ $t \leq 0$; by assumption $\left(A_{3}\right)$ we know that $u(r, 0)=\psi_{0}(r)=$ $\psi(r R(0), 0)$ is twice weakly differentiable on $[0,1], \psi_{0}^{\prime \prime} \in$ $L^{\infty}[0,1], \psi_{0}^{\prime}(0)=0$, and $\psi_{0}(1)=\sigma_{\infty}$.

Lemma 3. If the assumptions $\left(A_{1}\right)-\left(A_{3}\right)$ hold, then for any $T>0$ the problem (26), (20)-(25) has a unique solution $u_{\varepsilon}$ on $[0,1] \times[-\tau, T]$ satisfying the following assertions:

(i) $u_{\varepsilon}(y, t) \in W_{p}^{2,1}\left(\bar{Q}_{T}\right), p \in(1, \infty)$ and for any $\varepsilon>0$ there holds

$\left\|u_{\varepsilon}(|y|, t)\right\|_{W_{p}^{2,1}\left(\bar{Q}_{T}\right)} \leq C(T, p)\left(\left\|u_{0}(|y|, 0)\right\|_{W^{2, \infty}(0,1)}+\sigma_{\infty}\right)$,

where $C(T, p)$ is a constant independent of $\varepsilon, u_{0}(y, 0)=$ $\psi(z R(0), 0)$;

(ii) For any $\varepsilon>0$, there holds $0 \leq\left|u_{\varepsilon}(|y|, t)\right| \leq \sigma_{\infty}, 0 \leq$ $y \leq 1,-\tau \leq t \leq T$.

Remark 4. From (i) and assumption $\left(A_{2}\right)$ it is easy to get

$$
\begin{aligned}
& \left\|u_{\varepsilon}(|y|, t-\tau)\right\|_{W_{p}^{2,1}\left(\bar{Q}_{T}\right)} \\
& \quad \leq C(T, p)\left(\left\|u_{0}(|y|, 0)\right\|_{W^{2, \infty}(0,1)}+\sigma_{\infty}\right) .
\end{aligned}
$$

Proof. Endow $X_{T}$ with the following norm:

$$
\left\|\left(u, u_{\tau}\right)\right\|=\max _{[0,1] \times[-\tau, T]}|u|
$$

Then $X_{T}$ is clearly a Banach space. Define a mapping $F$ : $X_{T} \rightarrow X_{T}$ as follows: for any $\left(u, u_{\tau}\right) \in X_{T}$, define $F\left(u, u_{\tau}\right)=$ $\left(\tilde{u}, \tilde{u}_{\tau}\right)$, where $\tilde{u}(z, t)=\tilde{\widetilde{u}}\left(z y^{o}, t\right), y^{o} \in R^{3},\left|y^{o}\right|=1, u_{\tau}=$ $\tilde{u}(z, t-\tau)$, and $\widetilde{\widetilde{u}}$ is the solution of the following problem:

$$
\begin{gathered}
\frac{\partial \widetilde{\tilde{u}}}{\partial t}=d R_{0}^{-2} \exp \left\{-2 \int_{0}^{t} G_{\varepsilon}\left(u, u_{\tau}, \xi\right) d \xi\right\} \Delta \widetilde{\widetilde{u}} \\
\quad+G_{\varepsilon}\left(u, u_{\tau}, t\right) z \cdot \nabla \widetilde{\widetilde{u}} \\
\quad \widetilde{\widetilde{u}} f_{1}(u) H\left(u-\sigma_{N}\right), \\
\tilde{\widetilde{u}}(y, t)=\sigma_{\infty}, \quad|y|=1, t>0, \\
\frac{\partial \widetilde{\tilde{u}}}{\partial z}(z \rho(t)-0, t)=\frac{\partial \widetilde{\tilde{u}}}{\partial z}(z \rho(t)+0, t), \\
\quad 0 \leq z \leq 1, \quad t>0, \\
\tilde{\widetilde{u}}(z \rho(t)-0, t)=\tilde{\widetilde{u}}(z \rho(t)+0, t)=\sigma_{N}, \\
\tilde{\widetilde{u}}(y, t)=u_{0}(y, t), \quad|y| \leq 1,-\tau \leq t \leq 0,
\end{gathered}
$$

where $u_{0}(y, t)=\psi(z R(t), t)$. First we prove that $F$ is well defined. Since all coefficients in the first equation of (31) are bounded continuous functions and

$$
\begin{array}{r}
R_{0}^{-2} \exp \left\{-2 \int_{0}^{t} G_{\varepsilon}\left(u, u_{\tau}, \xi\right) d \xi\right\} \geq \lambda_{\varepsilon}\left(T,\left\|\left(u, u_{\tau}\right)\right\|, \tau\right)>0, \\
0 \leq t \leq T .
\end{array}
$$

Using the $L^{p}$ theory linear parabolic equations [29] we infer that the problem (31) has a unique solution $\widetilde{\widetilde{u}}$. Since the initial and boundary values are symmetric in space variable $y$, it is easy to see that, for any orthogonal transformation $O: R^{3} \rightarrow$ $R^{3}, \widetilde{\widetilde{u}}_{1}(y, t)=\tilde{\widetilde{u}}(O y, t)$ are also solutions of this problem which implies, by uniqueness of the solution, that there exists functions $\widetilde{u} \in C([0,1] \times[-\tau, T])$ such that

$$
\tilde{\widetilde{u}}(y, t)=\widetilde{u}(|y|, t), \quad|y| \leq 1,-\tau \leq t \leq T .
$$

By $L^{p}$ estimates we have, for $p \in(1, \infty)$,

$$
\begin{aligned}
\|\tilde{\widetilde{u}}(y, t)\| \leq & C(T, p,\|u\|)\left\|u_{0}(|y|, 0)\right\|_{W^{2, \infty}\left(B_{1}(0)\right)} \\
& +\sigma_{\infty}+\|\widetilde{\widetilde{u}}(|y|, t)\|_{L^{\infty}\left(\bar{Q}_{T}\right)} .
\end{aligned}
$$

By the maximum principle one can get

$$
0 \leq \tilde{\widetilde{u}}(y, t) \leq \sigma_{\infty}, \quad|y| \leq 1,0 \leq t \leq T
$$

Hence

$$
\begin{gathered}
\|\tilde{u}(|y|, t)\|_{W_{p}^{2,1}\left(\bar{Q}_{T}\right)} \\
\leq C\left(T, p,\left\|\left(u, u_{\tau}\right)\right\|\right) \\
\quad \times\left(\left\|u_{0}(|y|, 0)\right\|_{W^{2, \infty}(0,1)}+\sigma_{\infty}\right), \\
0 \leq \tilde{u}(y, t) \leq \sigma_{\infty}, \quad|y| \leq 1,-\tau \leq t \leq T .
\end{gathered}
$$

Taking particularly $p>(5 / 2)$, by the embedding $W_{p}^{2,1}\left(\bar{Q}_{T}\right) \hookrightarrow \hookrightarrow C\left(\bar{Q}_{T}\right),(p>(5 / 2))$, we infer that $\left(\tilde{u}, \tilde{u}_{\tau}\right) \in$ $X_{T}$. Hence the mapping $F$ is well defined.

Let $E=\left\{\left(u, u_{\tau}\right):\left(u, u_{\tau}\right) \in C([0,1] \times[0, T]) \times C([0,1] \times\right.$ $\left.[-\tau, T]), 0 \leq u, u_{\tau} \leq \sigma_{\infty}\right\}$. $E$ is clearly a bounded closed convex subset of $X_{T}$. We first prove that $F(E)$ is precontract in $X_{T}$. Actually, since, for any $\left(u, u_{\tau}\right) \in E,\left\|\left(u, u_{\tau}\right)\right\| \leq \sigma_{\infty}$ by (36) we infer that for any $1<p<\infty$ there hold

$$
\|\tilde{u}(|y|, t)\| \leq C(T, p) .
$$


Taking $p>(5 / 2)$ and using the compact embedding $W_{p}^{2,1}\left(\bar{Q}_{T}\right) \hookrightarrow \hookrightarrow C\left(\bar{Q}_{T}\right),(p>(5 / 2))$, we conclude that $F(E)$ is precontract in $X_{T}$.

Next we prove that $F$ is continuous. Let $\left(u_{1}, u_{1 \tau}\right)$, $\left(u_{2}, u_{2 \tau}\right) \in X_{T}$ and denote $\left(\widetilde{u}_{1}, \widetilde{u}_{1 \tau}\right)=F\left(u_{1}, u_{1 \tau}\right),\left(\widetilde{u}_{2}, \widetilde{u}_{2 \tau}\right)=$ $F\left(u_{2}, u_{2 \tau}\right), \widetilde{\widetilde{u}}^{*}=\widetilde{u}_{1}-\widetilde{u}_{2}, \widetilde{\tilde{u}}_{1 \tau}^{*}=\widetilde{u}_{1 \tau}-\widetilde{u}_{2 \tau}$. It is obvious that $\widetilde{\widetilde{u}}^{*}$ is a solution of the following problem:

$$
\begin{gathered}
\frac{\partial \tilde{\tilde{u}}^{*}}{\partial t}=d R_{0}^{-2} \exp \left\{-2 \int_{0}^{t} G_{\varepsilon}\left(u_{1}, u_{1 \tau}, \xi\right) d \xi\right\} \Delta \widetilde{\widetilde{u}}^{*} \\
+G_{\varepsilon}\left(u_{1}, u_{1 \tau}, t\right)\left(y \cdot \nabla \widetilde{\tilde{u}}^{*}\right) \\
-\widetilde{\tilde{u}}^{*} f\left(u_{1}\right) H_{\varepsilon}\left(u_{1}-\sigma_{N}\right)+h(y, t), \\
\tilde{\widetilde{u}}(y, t)=0, \quad|y|=1, t>0, \\
\frac{\partial \widetilde{\tilde{u}}}{\partial z}(z \rho(t)-0, t)=\frac{\partial \tilde{\tilde{u}}}{\partial z}(z \rho(t)+0, t), \\
0 \leq z \leq 1, \quad t>0, \\
\tilde{\tilde{u}}(z \rho(t)-0, t)=\tilde{\tilde{u}}(z \rho(t)+0, t)=0, \\
0 \leq z \leq 1, \quad t>0, \\
\tilde{\widetilde{u}}(y, t)=0, \quad|y| \leq 1,-\tau \leq t \leq 0,
\end{gathered}
$$

where $h(y, t)=d R_{0}^{-2}\left[\exp \left\{-2 \int_{0}^{t} G_{\varepsilon}\left(u_{1}, u_{1 \tau}, \xi\right) d \xi\right\}-\exp \{-2 \times\right.$ $\left.\left.\int_{0}^{t} G_{\varepsilon}\left(u_{2}, u_{2 \tau}, \xi\right) d \xi\right\}\right] \tilde{\tilde{u}}_{2}+\left[G_{\varepsilon}\left(u_{1}, u_{1 \tau}, t\right)-G_{\varepsilon}\left(u_{2}, u_{2 \tau}, t\right)\right](y$. $\left.\nabla \widetilde{\tilde{u}}_{2}\right)-\widetilde{\tilde{u}}_{2}\left[f_{1}\left(u_{1}\right) H_{\varepsilon}\left(u_{1}-\sigma_{N}\right)-f_{1}\left(u_{2}\right) H_{\varepsilon}\left(u_{2}-\sigma_{N}\right)\right]$.

Clearly,

$$
G_{\varepsilon}\left(u, u_{\tau}, t\right) \leq C\left(\left\|\left(u, u_{\tau}\right)\right\|, T\right)
$$

for $\left(u, u_{\tau}\right) \in X_{T}, t \in[0, T]$, where $C\left(\left\|\left(u, u_{\tau}\right)\right\|\right)$ is independent of $\varepsilon$. Then one can get

$e^{-2 T C\left(\left\|\left(u, u_{\tau}\right)\right\|, T\right)}$

$$
\begin{aligned}
& \leq \exp \left\{-2 \int_{0}^{t} G_{\varepsilon}\left(u, u_{\tau}, \xi\right)\right\} d \xi \leq e^{2 T C\left(\left\|\left(u, u_{\tau}\right)\right\|, T\right)}, \\
& \left|\exp \left\{-2 \int_{0}^{t} G_{\varepsilon}\left(u, u_{\tau}, \xi\right)\right\} d \xi-\exp \left\{-2 \int_{0}^{t^{\prime}} G_{\varepsilon}\left(u, u_{\tau}, \xi\right)\right\} d \xi\right| \\
& \leq C\left(\left\|\left(u, u_{\tau}\right)\right\|\right)\left|t-t^{\prime}\right|
\end{aligned}
$$

for $u, v \in X_{T}, t \in[0, T]$. Besides, it is also clear that

$$
0 \leq H_{\varepsilon}\left(u-\sigma_{N}\right) \leq 1, \quad|y| \leq 1,0 \leq t \leq T .
$$

Hence, by the $L^{p}$ estimate one can get

$$
\left\|\tilde{\tilde{u}}^{*}\right\|_{W_{p}^{2,1}\left(\overline{\mathrm{Q}}_{T}\right)} \leq C\left(T, p,\left\|\left(u_{1}, u_{1 \tau}\right)\right\|\right)\|h\|_{L^{p}\left(\overline{\mathrm{Q}}_{T}\right)} .
$$

By the fact that $H_{\varepsilon}$ is Lipschitz continuous and (36), one can verify

$$
\begin{aligned}
\left.\|h\|\right|_{L^{p}\left(\bar{Q}_{T}\right)} \leq & C\left(T, p,\left\|\left(u_{1}, u_{1 \tau}\right)\right\|,\left\|\left(u_{2}, u_{2 \tau}\right)\right\|, \varepsilon\right) \\
& \times\left\|\left(u_{1}-u_{2}, u_{1 \tau}-u_{2 \tau}\right)\right\| .
\end{aligned}
$$

Substituting these estimates into (43) we have

$$
\begin{aligned}
\left\|\tilde{\tilde{u}}^{*}\right\|_{W_{p}^{2,1}\left(\bar{Q}_{T}\right)} \leq & C\left(T, p,\left\|\left(u_{1}, u_{1 \tau}\right)\right\|,\left\|\left(u_{2}, u_{2 \tau}\right)\right\|, \varepsilon\right) \\
& \times\left\|\left(u_{1}-u_{2}, u_{1 \tau}-u_{2 \tau}\right)\right\| .
\end{aligned}
$$

Taking $p>(5 / 2)$ and using the embedding $W_{p}^{2,1}\left(\bar{Q}_{T}\right) \hookrightarrow \hookrightarrow$ $C\left(\overline{\mathrm{Q}}_{T}\right)$ we get

$$
\begin{aligned}
\|\left(\widetilde{u}_{1}\right. & \left.-\widetilde{u}_{2}, \widetilde{u}_{1 \tau}-\widetilde{u}_{2 \tau}\right) \| \\
& \leq\left\|\widetilde{\tilde{u}}^{*}\right\|_{W_{p}^{2,1}\left(\bar{Q}_{T}\right)} \\
& \leq C\left(T, p,\left\|\left(u, u_{\tau}\right)\right\|, \varepsilon\right)\left\|\left(u_{1}-u_{2}, u_{1 \tau}-u_{2 \tau}\right)\right\| .
\end{aligned}
$$

Hence, $F$ is continuous.

Since we have proven that $F$ maps $E$ into itself, using the Schauder fixed point theorem we conclude that $F$ has a fixed point in $E$. Therefore, the problem (19)-(25) has a solution $u=u_{\varepsilon}$.

Next, we prove uniqueness. Let $u^{*}=u_{1}-u_{2}$. Then $u^{*}$ satisfy

$$
\begin{aligned}
\frac{\partial u^{*}}{\partial t}= & d R_{0}^{-2} \exp \left\{-2 \int_{0}^{t} G_{\varepsilon}\left(u_{1}, u_{1 \tau}, \xi\right) d \xi\right\} \Delta u^{*} \\
& +G_{\varepsilon}\left(u_{1}, u_{1 \tau}, t\right)\left(y \cdot u^{*}\right)+p(y, t)
\end{aligned}
$$

for $|y|<1, t>0$, where $p(y, t)=d R_{0}^{-2}\left[\exp \left\{-2 \int_{0}^{t} G_{\varepsilon}\left(u_{1}, u_{1 \tau}\right.\right.\right.$, $\left.\xi) d \xi\}-\exp \left\{-2 \int_{0}^{t} G_{\varepsilon}\left(u_{2}, u_{2 \tau}, \xi\right) d \xi\right\}\right] \Delta u_{2}+\left[G_{\varepsilon}\left(u_{1}, u_{1 \tau}, t\right)-\right.$ $\left.G_{\varepsilon}\left(u_{2}, u_{2 \tau}, t\right)\right]\left(y \cdot \nabla u_{2}\right)-\left[f\left(u_{1}\right)-f\left(u_{2}\right)\right] H_{\varepsilon}\left(u_{1}(|y|, t)-\sigma_{N}\right)-$ $f\left(u_{2}\right)\left[H_{\varepsilon}\left(u_{1}(|y|, t)-\sigma_{N}\right)-H_{\varepsilon}\left(u_{2}(|y|, t)-\sigma_{N}\right)\right]$.

Multiplying (47) with $u^{*}$ and integrating in $y$, one can get

$$
\begin{aligned}
& \frac{1}{2} \int_{\|y\| \leq 1}\left(u^{*}(y, t)\right)^{2} d y \\
&=- d R_{0}^{-2} \exp \left\{-2 \int_{0}^{t} G_{\varepsilon}\left(u_{1}, u_{1 \tau}, \xi\right) d \xi\right\} \\
& \quad \int_{\|y\| \leq 1}\left|\nabla u^{*}(y, t)\right|^{2} d y \\
&-\frac{3}{2} G_{\varepsilon}\left(u_{1}, u_{1 \tau}, t\right) \int_{\|y\| \leq 1}\left(u^{*}(y, t)\right)^{2} d y \\
&+\int_{\|y\| \leq 1} p(y, t) u^{*}(y, t) d y \\
& \leq-d R_{0}^{-2} \exp \left(-2 T C\left(\left\|\left(u_{1}, u_{1 \tau}\right)\right\|\right)\right)\left\|\nabla u^{*}(\cdot, t)\right\|_{L^{2}\left(B_{1}(0)\right)}^{2} \\
&+\frac{3}{2} C\left(\left\|\left(u_{1}, u_{1 \tau}\right)\right\|\right)\left\|u^{*}(\cdot, t)\right\|_{L^{2}\left(B_{1}(0)\right)}^{2}+I(t),
\end{aligned}
$$


where $I(t)=I_{1}+I_{2}+I_{3}+I_{4}$ and

$$
\begin{gathered}
I_{1}=d R_{0}^{-2}\left[\exp \left\{-2 \int_{0}^{t} G_{\varepsilon}\left(u_{1}, u_{1 \tau}, \xi\right) d \xi\right\}\right. \\
\left.\quad-\exp \left\{-2 \int_{0}^{t} G_{\varepsilon}\left(u_{2}, u_{2 \tau}, \xi\right) d \xi\right\}\right] \\
\quad \times \int_{\|y\| \leq 1} \Delta u_{2} \cdot \nabla u^{*}(y, t) d y, \\
I_{2}=\left[G_{\varepsilon}\left(u_{1}, u_{1 \tau}, t\right)-G_{\varepsilon}\left(u_{2}, u_{2 \tau}, t\right)\right] \\
\quad \times \int_{\|y\| \leq 1}\left(y \cdot \nabla u_{2}\right) \nabla u^{*}(y, t) d y, \\
I_{3}=-\int_{\|y\| \leq 1}\left[f\left(u_{1}\right)-f\left(u_{2}\right)\right] H_{\varepsilon}\left(u_{1}(|y|, t)-\sigma_{N}\right) \\
\quad \times u^{*}(y, t) d y, \\
I_{4}=-\int_{\|y\| \leq 1} f\left(u_{2}\right)\left[H_{\varepsilon}\left(u_{1}(|y|, t)-\sigma_{N}\right)\right. \\
\left.-H_{\varepsilon}\left(u_{2}(|y|, t)-\sigma_{N}\right)\right] u^{*}(y, t) d y .
\end{gathered}
$$

By the mean value theorem and the fact that $f(u) \geq 0$, we have

$$
I_{4}=-\int_{\|y\| \leq 1} f\left(u_{2}\right) \int_{0}^{1} H_{\varepsilon}^{\prime} d \theta\left(u^{*}\right)^{2} d y \leq 0
$$

Using (28) and embedding $W_{p}^{2,1}\left(\bar{Q}_{T}\right) \hookrightarrow \hookrightarrow C\left(\bar{Q}_{T}\right),(p>$ $(5 / 2))$, we get

$$
\max _{\overline{\mathrm{Q}}_{T}}\left|\nabla u_{2}(|y|, t)\right| \leq C(T), \quad \max _{\overline{\mathrm{Q}}_{T}}\left|\nabla u_{2 \tau}(|y|, t)\right| \leq C(T) .
$$

From definition of $G_{\varepsilon}$ and Lipschitz continuous of $H_{\varepsilon}$, we have

$$
\left|G_{\varepsilon}\left(u_{1}, u_{1 \tau}, t\right)-G_{\varepsilon}\left(u_{2}, u_{2 \tau}, t\right)\right| \leq C(\varepsilon)\left(\left\|u^{*}\right\|_{2}+\left\|u_{\tau}^{*}\right\|_{2}\right) .
$$

Then by using (51) one can get

$$
I_{2} \leq C(\varepsilon)\left(\left\|u^{*}\right\|_{2}+\left\|u_{\tau}^{*}\right\|_{2}\right) \int_{\|y\| \leq 1}\left|u^{*}(y, t)\right| d y .
$$

By a direct computation and (52) one can get

$$
I_{1} \leq C(\varepsilon, T) \int_{0}^{t}\left(\left\|u^{*}\right\|_{2}+\left\|u_{\tau}^{*}\right\|_{2}\right) d \xi \int_{\|y\| \leq 1}\left|\nabla u^{*}(y, t)\right| d y .
$$

Lipschitz continuity of $f(u)$ combined with (51) yields

$$
I_{3} \leq C(\varepsilon, T) \int_{\|y\| \leq 1}\left|u^{*}(y, t)\right|^{2} d y .
$$

Summing up (51), (53)-(55) into $I(t)$, one can get

$$
\begin{aligned}
I(t) \leq & C(\varepsilon)\left(\left\|u^{*}\right\|_{2}+\left\|u_{\tau}^{*}\right\|_{2}\right) \int_{\|y\| \leq 1}\left|u^{*}(y, t)\right| d y \\
& +C(\varepsilon, T) \int_{0}^{t}\left(\left\|u^{*}\right\|_{2}+\left\|u_{\tau}^{*}\right\|_{2}\right) d \xi \int_{\|y\| \leq 1}\left|\nabla u^{*}(y, t)\right| d y \\
& +C(\varepsilon, T) \int_{\|y\| \leq 1}\left|u^{*}(y, t)\right|^{2} d y .
\end{aligned}
$$

Using Cauchy inequality and summing up (51), (53)-(55) into $I(t)$, one can get

$$
\begin{aligned}
\frac{1}{2} \frac{d}{d t}\left\|u^{*}(\cdot, t)\right\|_{2}^{2} \\
\leq-C_{1}(T)\left\|\nabla u^{*}(\cdot, t)\right\|_{2}^{2} \\
\quad+C_{2}\left\|u^{*}(\cdot, t)\right\|_{2}^{2}+C(\varepsilon)\left(\left\|u^{*}\right\|_{2}+\left\|u_{\tau}^{*}\right\|_{2}\right) \\
\quad \times \int_{\|y\| \leq 1}\left|u^{*}(y, t)\right| d y \\
\quad+C(\varepsilon, T) \int_{0}^{t}\left(\left\|u^{*}\right\|_{2}+\left\|u_{\tau}^{*}\right\|_{2}\right) d \xi \int_{\|y\| \leq 1}\left|\nabla u^{*}(y, t)\right| d y \\
\quad+C(\varepsilon, T) \int_{\|y\| \leq 1}\left|u^{*}(y, t)\right|^{2} d y .
\end{aligned}
$$

Denoting $w(t)=\left\|u^{*}(\cdot, t)\right\|_{2}^{2}$ and $w_{1}(t)=\left\|\nabla u^{*}(\cdot, t)\right\|_{2}^{2}$, one can get

$$
\begin{aligned}
\frac{1}{2} \frac{d w}{d t} \leq & -C_{1}(T) w_{1}(t)+C_{2}(T) w(t) \\
& +J(t)+H(t)+C(\varepsilon, T) w(t),
\end{aligned}
$$

where $J(t)=C(\varepsilon)\left(\left\|u^{*}\right\|_{2}+\left\|u_{\tau}^{*}\right\|_{2}\right) \int_{\|y\| \leq 1}\left|u^{*}(y, t)\right| d y=$ $C(\varepsilon)[\sqrt{w(t)}+\sqrt{w(t-\tau)}] \sqrt{w(t)}, H(t)=C(\varepsilon, T) \int_{0}^{t}\left(\left\|u^{*}\right\|_{2}+\right.$ $\left.\left\|u_{\tau}^{*}\right\|_{2}\right) d \xi \int_{\|y\| \leq 1}\left|\nabla u^{*}(y, t)\right| d y=C(\varepsilon, T) \int_{0}^{t} \sqrt{w(t)}+$ $\sqrt{w(t-\tau)} d \xi \sqrt{w_{1}(t)}$. By the fact that $u^{*}=0$, for $-\tau \leq t \leq 0$, then

$$
\begin{aligned}
w(t-\tau) & =\left\|u^{*}(\cdot, t-\tau)\right\|_{2}^{2}=\int_{\|y\| \leq 1, t>0}\left|u^{*}(\cdot, t-\tau)\right|^{2} d y \\
& =\int_{\|y\| \leq 1, t>0}\left|u^{*}(\cdot, t)\right|^{2} d y+\int_{\|y\| \leq 1,-\tau \leq t \leq 0}\left|u^{*}(\cdot, t)\right|^{2} d y \\
& =\int_{\|y\| \leq 1, t>0}\left|u^{*}(\cdot, t)\right|^{2} d y=w(t)=\left\|u^{*}(\cdot, t)\right\|_{2}^{2} .
\end{aligned}
$$

It follows that

$$
J(t) \leq 2 C(\varepsilon) w(t)
$$

$$
H(t) \leq C(\varepsilon, T) C(\delta) \int_{0}^{t} w(\xi) d \xi+C(\varepsilon, T) \delta w_{1}(t),
$$


where we have used the $\delta$-Cauchy inequality, $\delta$ is an arbitrary positive number, and $C(\delta)$ is a positive constant depending on $\delta$. Then we have

$$
\begin{aligned}
\frac{1}{2} \frac{d w}{d t} \leq & -C_{1}(T) w_{1}(t)+C_{3}(T) w(t) \\
& +2 C(\varepsilon) C(\delta) \int_{0}^{t} w(\xi) d \xi+C(\varepsilon, T) \delta w_{1}(t)
\end{aligned}
$$

where $C_{3}(T)=C_{2}(T)+2 C(\varepsilon)+C(\varepsilon, T)$. Therefore, by choosing $\delta$ sufficiently small such that $C(\varepsilon, T) \delta<C_{1}(T)$ one can get

$$
\frac{1}{2} \frac{d w}{d t} \leq C_{3}(T) w(t)+2 C(\varepsilon) C(\delta) \int_{0}^{t} w(\xi) d \xi
$$

Since $w(0)=0$, by Gronwall lemma we conclude that $w(t) \equiv$ 0 , for $0 \leq t \leq T$. Hence $u_{1}=u_{2}$.

Lemma 5. If conditions $\left(A_{1}\right)-\left(A_{3}\right)$ are satisfied, then for any $T>0$ the problem (19)-(25) has a unique solution $\left(u, u_{\tau}\right)$ on $X_{T}$ satisfying

$$
\begin{gathered}
u(|y|, t), u(|y|, t-\tau) \in W_{p}^{2,1}\left(\bar{Q}_{T}\right), \quad p \in(1, \infty) \\
0 \leq u(z, t) \leq \sigma_{\infty}, \quad 0 \leq z \leq 1,-\tau \leq t \leq T
\end{gathered}
$$

Proof. By Lemma 3 for any $\varepsilon>0$ the problem (26), (20)(25) has a unique solution $u_{\varepsilon}$ on $[0,1] \times[-\tau, T]$. Take a number $p>5$, by compact embedding $W_{p}^{2,1}\left(\bar{Q}_{T}\right) \hookrightarrow \hookrightarrow$ $C^{\alpha,(\alpha / 2)}\left(\bar{Q}_{T}\right)(0<\alpha<2-(5 / p))$. It follows that one can find a sequence of positive numbers $\varepsilon_{k} \rightarrow 0, k \rightarrow \infty$, and function $u \in W_{p}^{2,1}\left(\bar{Q}_{T}\right)$ such that if we denote $u_{k}=u_{\varepsilon_{k}}$, then

$$
\begin{aligned}
& u_{k}(|y|, t) \rightarrow u(|y|, t), \nabla u_{k}(|y|, t) \rightarrow \nabla u(|y|, t) \\
& \text { uniformly for }(y, t) \in \bar{Q}_{T}, \\
& u_{k}(|y|, t-\tau) \rightarrow u(|y|, t-\tau), \nabla u_{k}(|y|, t-\tau) \rightarrow \\
& \nabla u(|y|, t-\tau) \text { uniformly for }(y, t) \in \bar{Q}_{T}, \\
& u_{k t}(|y, t|) \vec{w} u_{t}(|y|, t)\left(L^{p}\left(\bar{Q}_{T}\right)\right),\left(\partial^{2} u_{k} / \partial y_{i} \partial y_{j}\right) \vec{w}\left(\partial^{2} u\right. \\
& \left./ \partial y_{i} \partial y_{j}\right)\left(L^{p}\left(\bar{Q}_{T}\right)\right) .
\end{aligned}
$$

Clearly $\left\{H_{\varepsilon k}\left(u-\sigma_{N}\right)\right\}$ is a bounded sequence in $L^{\infty}\left(\bar{Q}_{T}\right)$, so we can find a subsequence of $\left\{\varepsilon_{k}\right\}$ which is still denoted as $\left\{\varepsilon_{k}\right\}$ and a function $h \in L^{\infty}\left(\bar{Q}_{T}\right)$, such that

$$
\begin{gathered}
H_{\varepsilon k}\left(u-\sigma_{N}\right) \vec{w} h\left(L^{\infty}\left(\bar{Q}_{T}\right)\right), \\
G_{\varepsilon k}\left(u_{\varepsilon_{k}}, u_{\tau \varepsilon_{k}}, t\right) \overrightarrow{* w} m(t)\left(L^{\infty}\left(\bar{Q}_{T}\right)\right),
\end{gathered}
$$

where

$$
\begin{gathered}
m(t)=s\left(\int_{0}^{1} \frac{R_{\tau}^{3}}{R^{3}} u_{\tau} h(z, t-\tau) z^{2}-\widetilde{\sigma} h(z, t) z^{2}\right. \\
\left.-\lambda[1-h(z, t)] z^{2} d z\right) .
\end{gathered}
$$

Taking $\varepsilon=\varepsilon_{k}$ in (26), replacing $u, u_{\tau}$ with $u_{k}, u_{\tau k}$, respectively, and letting $k \rightarrow \infty$, one can get

$$
\begin{gathered}
\frac{\partial u}{\partial t}=d R_{0}^{-2} \exp \left\{-2 \int_{0}^{t} m(\xi) d \xi\right\} \frac{1}{z^{2}} \frac{\partial}{\partial z}\left(z^{2} \frac{\partial u}{\partial z}\right) \\
+m(t) z u_{z}-f(u) h, \\
0<z<1, \quad t>0, \\
\frac{\partial u}{\partial z}(0, t)=0, \quad u(1, t)=\sigma_{\infty}, \quad t>0 \\
u(z, t)=\psi(z R(t), t), \quad 0 \leq z \leq 1,-\tau \leq t \leq 0 \\
\frac{\partial u}{\partial z}(z \rho(t)-0, t)=\frac{\partial u}{\partial z}(z \rho(t)+0, t), \quad 0 \leq z \leq 1, \quad t>0 \\
u(z \rho(t)-0, t)=u(z \rho(t)+0, t)=\sigma_{N}, \quad 0 \leq z \leq 1, t>0 \\
\rho(t)=\phi(t), \quad-\tau \leq t \leq 0, \\
R(t)=\varphi(t), \quad-\tau \leq t \leq 0 .
\end{gathered}
$$

We assert that

$$
h(|y|, t)=\left\{\begin{array}{ll}
1, & u(|y|, t)>\sigma_{N}, \\
0, & u(|y|, t)<\sigma_{N},
\end{array} \quad\right. \text { a.e. }
$$

Actually, it is easy to verify that, for any $\delta>0, h(|y|, t)=1$ for a.e. $\left.(y, t) \in\left\{(y, t) \in \bar{Q}_{T}\right): u(|y|, t) \geq \sigma_{N}+\delta\right\}$. By the arbitrariness of $\delta$, we infer that $h(|y|, t)=1$ for a.e. $(y, t) \epsilon$ $\left.\left\{(y, t) \in \bar{Q}_{T}\right): u(|y|, t)>\sigma_{N}\right\}$. Similarly we can prove that $h(|y|, t)=0$ for a.e. $(y, t) \in\left\{(y, t) \in \bar{Q}_{T}: u(|y|, t)<\sigma_{N}\right\}$. This proves the assertion

Since $u_{t}=0, \nabla u=0$, and $\Delta u=0$ a.e. on the set $\{(y, t) \in$ $\left.\bar{Q}_{T}: u(|y|, t)=\sigma_{N}\right\}$, by (66) it follows that $h=0$ a.e. on the set $\left\{(y, t) \in \bar{Q}_{T}: u(|y|, t)=\sigma_{N}\right\}$. Hence

$$
h(|y|, t)=H\left(u(|y|, t)-\sigma_{N}\right), \quad(y, t) \in \bar{Q}_{T} .
$$

Substituting (74) into (65), one can get

$$
m(t)=G\left(u, u_{\tau}, t\right) .
$$

By (66), (74), and (75) we conclude that $u=u(z, t)$ is a solution of (19)-(25). This proves the existence of a solution. The rest of the assertions of Lemma 5 follow immediately from taking the weak limit in (26).

By Lemmas 2 and 5, we see that Theorem 1 immediately follows.

\section{Conclusions}

In this paper a mathematical model for the growth of necrotic tumors with time delays in proliferation is studied. By transforming this problem into an initial-boundary value problem in fixed domain of a coupled system of a parabolic equation 
and one integrodifferential equation with time delays, in which all equations involve discontinuous terms, and using the approximation method combined with Schauder fixed point theorem, we prove that this problem has a unique global solution in any time interval $[0, T]$.

The model we study in this paper is established by modifying the model studied in [15] by introducing a time delay in proliferation as that in Byrne [1]. It should be pointed out that, in [15], the authors only studied the existence and uniqueness of stationary solutions to the model (2)-(4), but in our study we mainly discuss the existence and uniqueness of the solution to the model (2)-(4) under some assumptions.

\section{Conflict of Interests}

The authors declare that there is no conflict of interests regarding the publication of this paper.

\section{Acknowledgments}

The authors express their thanks to anonymous referees for their careful comments and valuable suggestions on modification of the original paper. This work is supported by the NSF of China (11226182, 11301474, and 11171295) and the Foundation for Distinguished Young Teacher in Higher Education of Guangdong, China (Yq2013163).

\section{References}

[1] H. M. Byrne, "The effect of time delays on the dynamics of avascular tumor growth," Mathematical Biosciences, vol. 144, no. 2, pp. 83-117, 1997.

[2] H. M. Byrne and M. Chaplain, "Growth of nonnecrotic tumors in the presence and absence of inhibitors," Mathematical Biosciences, vol. 130, no. 2, pp. 151-181, 1995.

[3] H. Byrne and M. Chaplain, "Growth of necrotic tumors in the presence and absence of inhibitors," Mathematical Biosciences, vol. 135, no. 2, pp. 187-216, 1996.

[4] H. M. Byrne, T. Alarcon, M. R. Owen, S. D. Webb, and P. K. Maini, "Modelling aspects of cancer dynamics: a review," Philosophical Transactions of the Royal Society of London A, vol. 364, no. 1843, pp. 1563-1578, 2006.

[5] R. Eftimie, J. L. Bramson, and D. J. D. Earn, "Interactions between the immune system and cancer: a brief review of nonspatial mathematical models," Bulletin of Mathematical Biology, vol. 73, no. 1, pp. 2-32, 2011.

[6] H. Greenspan, "Models for the growth of solid tumor by diffusion," Studies in Applied Mathematics, vol. 51, pp. 317-340, 1972.

[7] H. P. Greenspan, "On the growth and stability of cell cultures and solid tumors," Journal of Theoretical Biology, vol. 56, no. 1, pp. 229-242, 1976.

[8] J. D. Nagy, "The ecology and evolutionary biology of cancer: a review of mathematical models of necrosis and tumor cell diversity," Mathematical Biosciences and Engineering, vol. 2, no. 2, pp. 381-418, 2005.

[9] M. J. Piotrowska, "Hopf bifurcation in a solid avascular tumour growth model with two discrete delays," Mathematical and Computer Modelling, vol. 47, no. 5-6, pp. 597-603, 2008.
[10] R. R. Sarkar and S. Banerjee, "A time delay model for control of malignant tumor growth," in Proceedings of the National Conference on Nonlinear Systems and Dynamics, pp. 1-4, 2006.

[11] K. E. Thompson and H. M. Byrne, "Modelling the internalization of labelled cells in tumour spheroids," Bulletin of Mathematical Biology, vol. 61, no. 4, pp. 601-623, 1999.

[12] J. Ward and J. King, "Mathematical modelling of avasculartumor growth II: modelling growth saturation," IMA Journal of Mathematics Applied in Medicine and Biology, vol. 15, pp. 1-42, 1998.

[13] U. Foryś and A. Mokwa-Borkowska, "Solid tumour growth analysis of necrotic core formation," Mathematical and Computer Modelling, vol. 42, no. 5-6, pp. 593-600, 2005.

[14] M. Bodnar and U. Foryś, "Time delay in necrotic core formation," Mathematical Biosciences and Engineering, vol. 2, no. 3, pp. 461-472, 2005.

[15] H. Bueno, G. Ercole, and A. Zumpano, "Stationary solutions of a model for the growth of tumors and a connection between the nonnecrotic and necrotic phases," SIAM Journal on Applied Mathematics, vol. 68, no. 4, pp. 1004-1025, 2008.

[16] S. Cui, "Formation of necrotic cores in the growth of tumors: analytic results," Acta Mathematica Scientia B, vol. 26, no. 4, pp. 781-796, 2006.

[17] S. Cui and A. Friedman, "Analysis of a mathematical model of the effect of inhibitors on the growth of tumors," Mathematical Biosciences, vol. 164, no. 2, pp. 103-137, 2000.

[18] S. B. Cui, "Analysis of a free boundary problem modeling tumor growth," Acta Mathematica Sinica, vol. 21, no. 5, pp. 1071-1082, 2005.

[19] S. Cui and S. Xu, "Analysis of mathematical models for the growth of tumors with time delays in cell proliferation," Journal of Mathematical Analysis and Applications, vol. 336, no. 1, pp. 523-541, 2007.

[20] U. Fory and M. Bodnar, "Time delays in proliferation process for solid avascular tumour," Mathematical and Computer Modelling, vol. 37, no. 11, pp. 1201-1209, 2003.

[21] X. M. Wei and S. B. Cui, "Existence and uniqueness of global solutions of a free boundary problem modeling tumor growth," Acta Mathematica Scientia A, vol. 26, no. 1, pp. 1-8, 2006 (Chinese).

[22] S. Xu, M. Bai, and X. Q. Zhao, "Analysis of a solid avascular tumor growth model with time delays in proliferation process," Journal of Mathematical Analysis and Applications, vol. 391, no. 1, pp. 38-47, 2012.

[23] P. Macklin and J. Lowengrub, "Nonlinear simulation of the effect of microenvironment on tumor growth," Journal of Theoretical Biology, vol. 245, no. 4, pp. 677-704, 2007.

[24] B. Zubik-Kowal, "Numerical algorithm for the growth of human tumor cells and their responses to therapy," Applied Mathematics and Computation, vol. 230, pp. 174-179, 2014.

[25] A. Friedman and F. Reitich, "Analysis of a mathematical model for the growth of tumors," Journal of Mathematical Biology, vol. 38, no. 3, pp. 262-284, 1999.

[26] S. Xu, "Analysis of a delayed free boundary problem for tumor growth," Discrete and Continuous Dynamical Systems B, vol. 15, no. 1, pp. 293-308, 2011.

[27] C. Bellomo, "A mathematical model of the immersion of a spherical tumor with a necrotic core into a nutrient bath," Mathematical and Computer Modelling, vol. 43, no. 7-8, pp. 779786, 2006. 
[28] W. Hao, J. D. Hauenstein, B. Hu, Y. Liu, A. J. Sommese, and Y.T. Zhang, "Bifurcation for a free boundary problem modeling the growth of a tumor with a necrotic core," Nonlinear Analysis. Real World Applications, vol. 13, no. 2, pp. 694-709, 2012.

[29] Z. Wu, J. Yin, and C. Wang, Elliptic and Parabolic Equations, China Science Press, Beijing, China, (Chinese). 


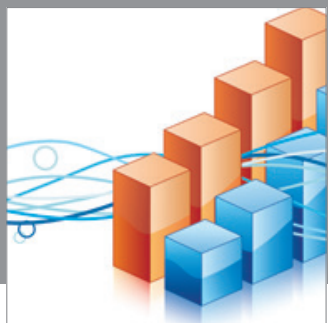

Advances in

Operations Research

mansans

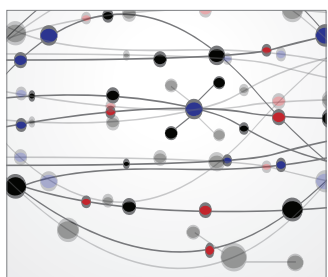

The Scientific World Journal
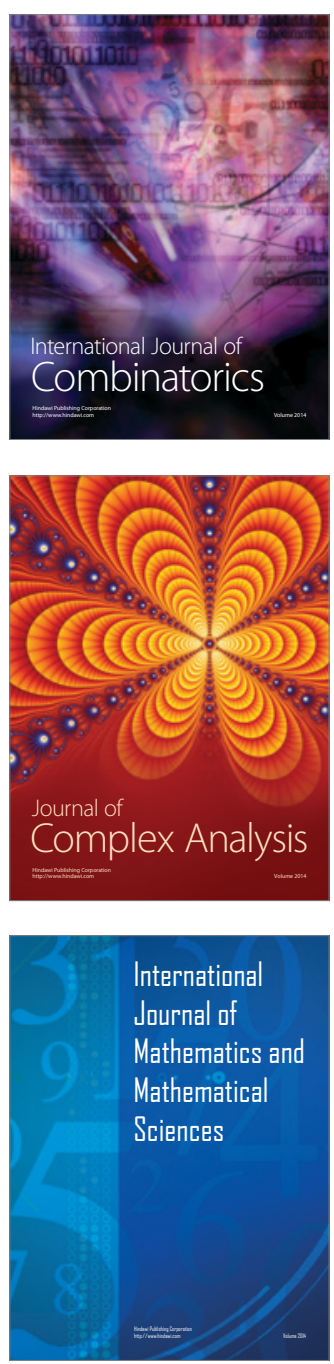
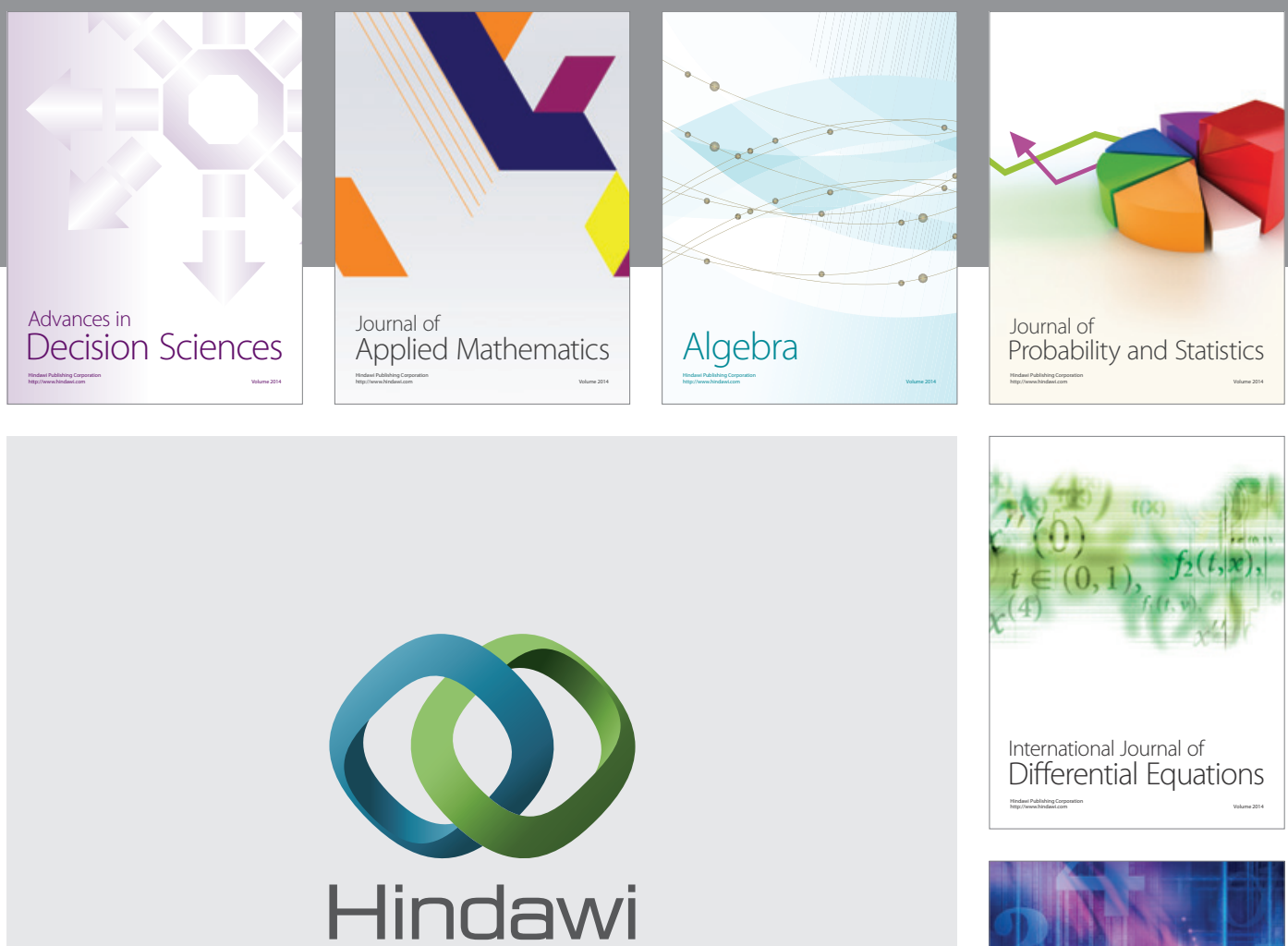

Submit your manuscripts at http://www.hindawi.com
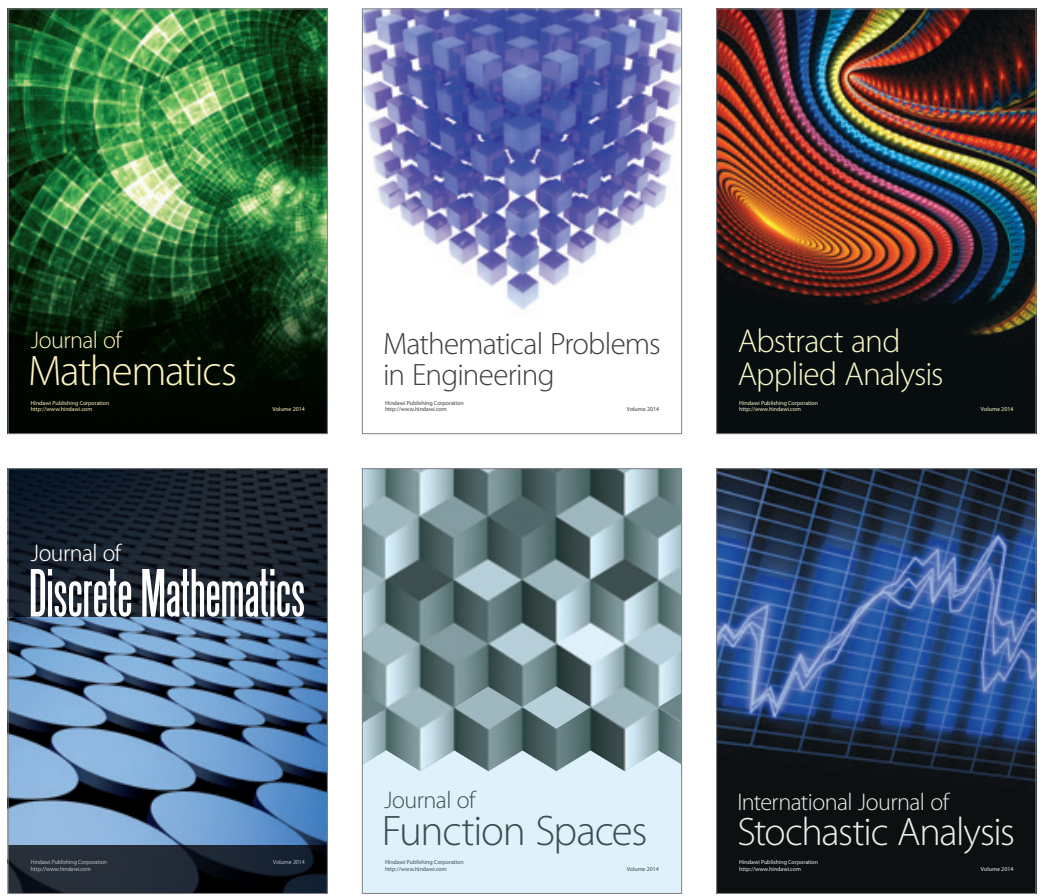

Journal of

Function Spaces

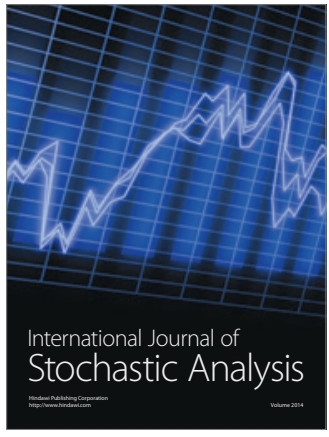

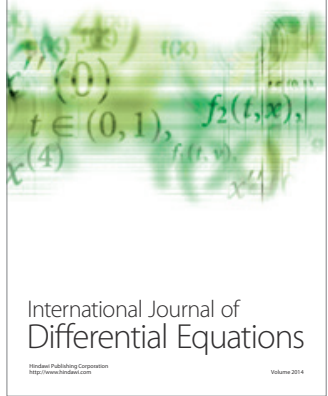
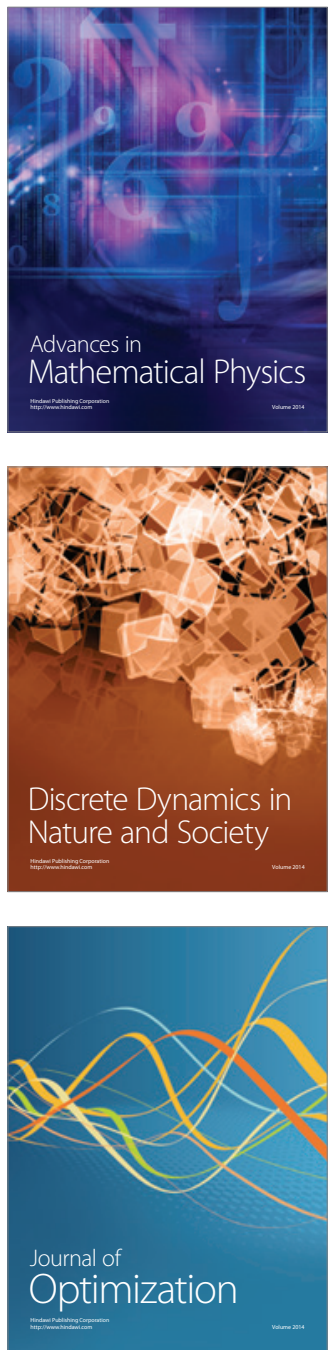